\title{
Evaluación de la peligrosidad con criterios geomorfológicos a través de las unidades de diagnóstico en el territorio de Casapalma (Valle de Guadalhorce, España)
}

\author{
Jesús RodRIGo COMINO \\ Departamento de Geografía \\ Universidad de Málaga \\ geo.jrc@gmail.com \\ Emilio FERRE BUENO \\ Departamento de Geografía \\ Universidad de Málaga \\ José María SENCIALES GONZÁLEZ \\ Departamento de Geografía \\ Universidad de Málaga
}

Recibido: 29 de abril del 2014

Enviado a evaluar: 8 de mayo del 2014

Aceptado: 24 de junio del 2014

\section{RESUMEN}

El presente trabajo muestra los resultados de una metodología combinada para la zonificación de la peligrosidad con orígenes geomorfológicos sobre un territorio. Para este propósito, se han utilizado como herramientas principales las unidades de diagnóstico y las técnicas de evaluación multicriterio con Sistemas de Información Geográfica (SIG). Normalmente estas técnicas no han sido aplicadas en el medio mediterráneo. Por lo tanto, para este caso, se ha llevado a cabo como ejemplo un estudio en el área del Valle del Guadalhorce (provincia de Málaga, España) con un paisaje marcado por un sistema de terrazas fluviales y glacis. Con dichas técnicas, se han obtenido cinco categorías de peligrosidad con origen geomorfológico (1=muy poca; $5=$ muy alta). De las 1872.68 ha del área de estudio, el $51.93 \%$ se encuentra en intervalos de peligrosidad alta debido a: (1) problemas de arroyada concentrada en las laderas; (2) actividad erosiva espasmódica del cauce principal y sus afluentes; (3) inestabilidad por movimientos en masa de áreas compuestas por arcillas expansivas con procesos vérticos.

Palabras clave: Peligrosidad, geomorfología, unidades de diagnóstico, evaluación multicriterio, SIG. 


\title{
Dangerousness evaluation with geomorphological criteria through diagnostic units in Casapalma territory (Gualhorce Valley, Spain)
}

\begin{abstract}
This paper presents the results of a combined methodology for zoning the dangerousness with geomorphological origins on a territory. For this purpose, diagnostic units and techniques of multicriteria analysis with Geographic Information Systems (GIS) are used as main tools for the analysis. Usually these types of studies are not applied with this methodology in the Mediterranean environment. Thus, in this case, this application is carried out as example in one part of the Guadalhorce Valley (Province of Malaga) with fluvial terraces and glacis landscapes. In this way, using this methodological sequence, five categories of dangerousness $(1=$ very low; $5=$ very high $)$ are obtained. With 1872.68 ha, $51.93 \%$ has high dangerousness with geomorphological origins. This situation is caused by: (1) concentrate runoff problems; (2) spasmodic erosive activity of the main stream and its tributaries; (3) landslides in areas with expansive clays and vertic processes.
\end{abstract}

Key words: Dangerousness, geomorphology, diagnostic units, multi-criteria analysis, GIS.

\section{Critéres d'evaluation des risques avec géomorphologique a travers des unites de diasnostic dans le territoire de Casapalma (Guadalhorce Valley, Espagne)}

\section{RÉSUMÉ}

Cet article présente les résultats d'une combinée méthodologie pour zonage de territoire avec niveaux de danger avec origines géomorphologiques. À cette fin, ont été utilisés comme les principaux des unités de diagnostic et techniques d'évaluation multi-critères avec les Systèmes d'Information Géographiques (SIG). Habituellement, ces types d'études ne sont pas appliqués avec cette méthodologie dans l'environnement méditerranéen. Par conséquent, dans ce cas, il a été considéré, par exemple, une étude dans la zone de Valle del Guadalhorce (Málaga, Espagne) avec un paysage marqué par un système de terrasses fluviales et glacis. Avec ces techniques, on a obtenu cinq catégories de danger avec l'origine géomorphologique $(1=$ très peu, $5=$ très élevé). With 1872.68 ha, $51.93 \%$ has high dangerousness with geomorphological origins. Avec 1872,68 ha, 51,93\% ont une forte dangerosité avec des origines géomorphologiques. Cette situation est causée par: (1) se concentrer problèmes de ruissellement; (2) L'activité érosive spasmodique du courant principal et de ses affluents; (3) glissements de terrain dans les zones à argiles gonflantes et les processus vertic.

Mots clés: Danger, géomorphologie, unités de diagnostic, analyse multi-critères, SIG.

\section{INTRODUCCIÓN}

La planificación territorial que pretende ordenar y estructurar un espacio geográfico debe focalizar sus estudios en el conocimiento del territorio que se va a gestionar. Este se basa en múltiples parámetros, decisiones e informaciones de disciplinas que trabajan dentro del marco socio-económico, los bienes culturales o las ciencias de la Tierra. En este último grupo, la Geomorfología aporta una base considerable al conocimiento de las características físicas y morfológicas del territorio (Panizza et al., 1987).

De esta forma, la cartografía geoambiental con criterios fisiográficos constituye una base científica para la planificación a corto, medio y largo plazo, ya que 
representa los distintos rasgos constituyentes de la superficie terrestre y los procesos que actúan sobre la misma (Peña Monné, 1997). Dentro de ella, los mapas de unidades de diagnóstico responden a esta necesidad, proporcionando una base científica para la ordenación del territorio a través de la delimitación de espacios geomorfológicos homogéneos (Zinck, 2012).

Esta cartografía tiene su origen en el conocido trabajo de "Lands systems" del C.S.I.R.O. australiano (Commonwealth Scientific and Industrial Research Organisation) y en el denominado "método geosistémico para el análisis geointegrado del paisaje" (Bertrand y Bertrand, 2006). Estas tentativas las continuaron los investigadores del ITC (Faculty of Geo-Information Science and Earth Observation de Enschede, Países Bajos) con la combinación de parámetros geomorfológicos (van Zuidam y van Zuidam-Cancelado, 1979; Verstappen y van Zuidam, 1991; Zinck, 2012).

Para ello, los SIG (Sistemas de Información Geográfica) se predisponen como una herramienta principal junto con los conocimientos del experto para generar una cartografía apropiada, a través de la superposición y tratamiento de capas en formato ráster y vectorial (Van Westen et al., 2003). Además, numerosos autores para precisar sus resultados y crear modelizaciones de realidades que no han ocurrido aún, también los han combinado con complejos aparatos estadísticos como las evaluaciones multicriterio (Feizizadehab y Blaschkeb, 2013). Este método permite resolver con "todo rigor la interrelación de las diversas variables del territorio, otorgándosele el peso y la proporcionalidad adecuada o necesaria en cada momento" (Galacho Jiménez y Arrebola Castaño, 2013).

Sin embargo, a pesar de los estudios realizados a través de esta contrastada metodología de la evaluación multicriterio aunada con los SIG, todavía no se ha alcanzado un consenso sobre qué variables geomorfológicas y geológicas específicas en el Mediterráneo deben utilizarse para la generación de estos mapas (Verstappen y van Zuidam, 1991; Tapia-Varela y López-Blanco, 2004; Castillo Rodríguez et al. 2010). En relación con esta combinación metodológica y el presente trabajo, se encuentran aquellos estudios que toman el medio natural como objeto de experimentación para la gestión del territorio (Paegelow et al., 2004; Camacho et al., 2007; Galacho Jiménez y Arrebola Castaño, 2008-a, 2008-b; Feizizadehab y Blaschkeb, 2013).

Así, los objetivos de este trabajo en relación a lo anteriormente comentado son: i) la proposición de acertadas variables que condicionan un medio mediterráneo y, más concretamente, las específicas para un área enmarcada dentro de un valle fluvial; ii) conjugar dicha información en un mapa de unidades de diagnóstico; iii) readaptar las técnicas de evaluación multicriterio y SIG para el estudio de la peligrosidad de un medio mediterráneo con criterios geomorfológicos.

De esta forma, al final de este trabajo se mostrarán claves precisas para ayudar al gestor del territorio a señalar con exactitud qué áreas deben ser catalogadas como peligrosas y cuáles como estables en diferentes intervalos, representado todo en un solo mapa. 


\section{2. ÁREA DE ESTUDIO}

El espacio escogido está integrado dentro del tramo medio del río Guadalhorce (provincia de Málaga, España) con un área total de $18.72 \mathrm{~km}^{2}$ (Figura 1). Este curso de agua es el más importante de la provincia en cuanto a volumen de caudal movilizado (alrededor de $450 \mathrm{l} / \mathrm{s}$ ) y con un recorrido de $154 \mathrm{~km}$. Tiene un trazado E$\mathrm{W}$ en su inicio y, N-S y W-E a medida que se acerca a la costa. Desemboca en una zona con gran contenido de arenas y aguas salinas con dificultad de drenaje en el mar de Alborán al oeste de la ciudad de Málaga. Posee un carácter eminentemente calcáreo que condiciona la calidad del agua en algunos tramos y está controlado por el conjunto de embalses de "El Chorro" (Sanz de Galdeano y López Garrido, 2013).

Figura 1. Mapa de localización del área de estudio.

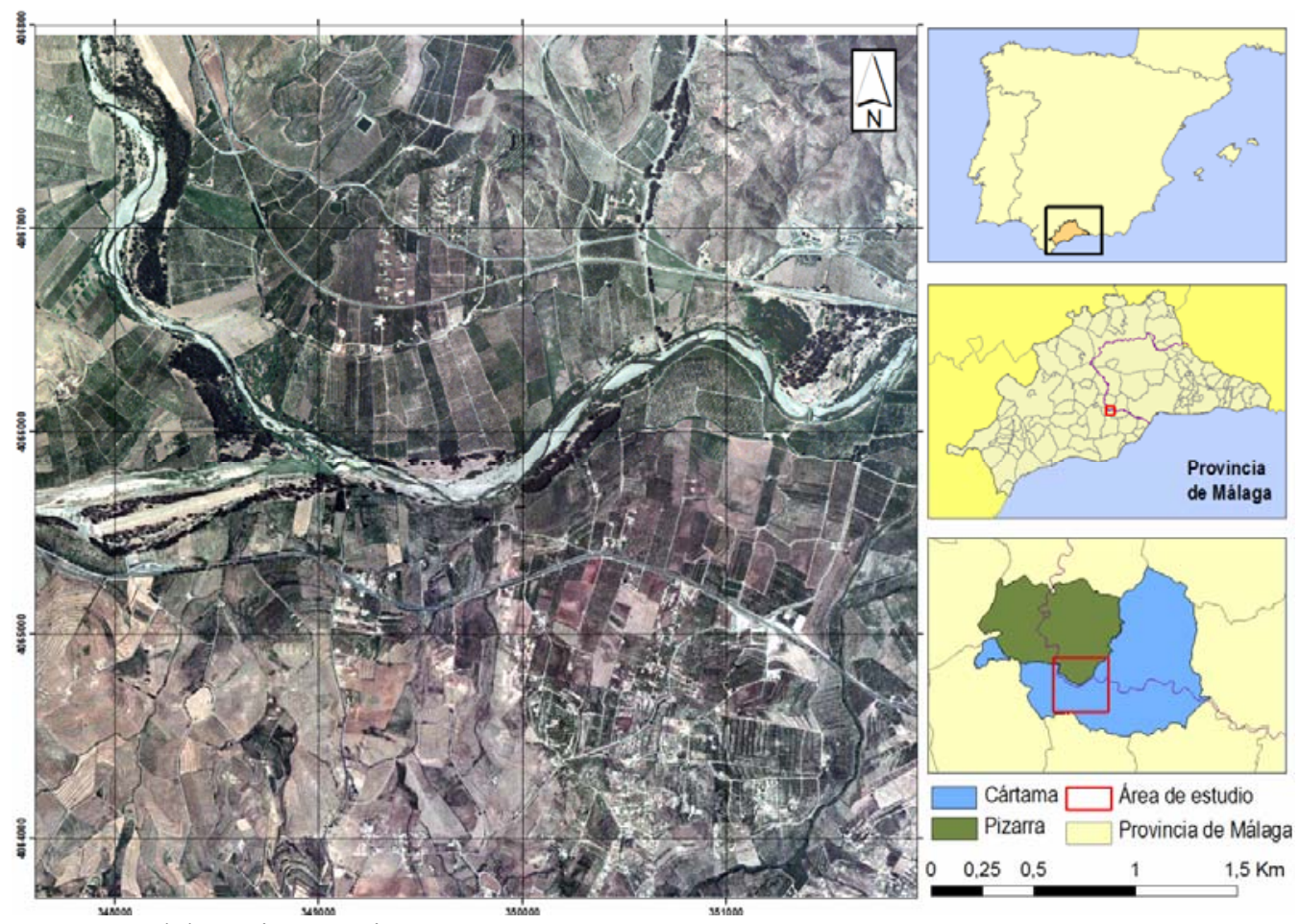

Fuente: Elaboración propia.

La zona de estudio se localiza cerca del tramo final en la unidad fisiográfica conocida como Hoya de Málaga en los términos administrativos de los municipios de Cártama y Pizarra, concretamente, en un área conocida como Casapalma. Existe un intensivo aprovechamiento agrícola por los suelos profundos y bien estructurados que se han formado de tipo fluvisol y cambisol (Aguilar Ruiz et al., 1994; Rodrigo Comino, 2012) en formas deposicionales de tipo glacis y sistemas de terrazas 
fluviales. En ocasiones, estas morfologías son desestabilizadas por la construcción de infraestructuras, núcleos urbanos dispersos y cultivos de cítricos de carácter intensivo.

\section{MÉTODOS Y FUENTES}

En un primer paso, se propone la utilización de variables geomorfológicas acordes con las características del medio mediterráneo para la realización de una cartografía de base: pendientes, asociaciones litológicas, morfologías y sistemas de paisaje (Ferre Bueno, 1997-a, 1997-b; Panizza, 2005). Estos mapas fueron digitalizados en una fase preliminar en formato vectorial, y contrastados con observaciones de campo y fotointerpretación con ayuda de modelos en 3D elaborados a partir del módulo ArcScene de ArcGis 10.1 y el software ER Mapper 7.0 de teledetección. Además, se traslada la información desde las diferentes fuentes geológicas (IGME, 1978) y edáficas (Aguilar Ruiz et al., 1994) presentadas a escala $1 / 50000$ hasta $1 / 25000$ que existen en el territorio.

A continuación, son cruzados los resultados de cada mapa a través de técnicas de superposición y eliminados los polígonos residuales (con las herramientas de edición de capas vectoriales de ArcGis 10.1), para obtener uno solo que será denominado como de "unidades de diagnóstico" (van Zuidam y van Zuidam-Cancelado, 1979; Verstappen y van Zuidam, 1991; Zinck, 2012). A partir de aquí, en un segundo bloque de trabajo, se realiza un inventario de cada unidad a través del trabajo de campo y la fotointerpretación de: sistemas morfogenéticos, marcas de erosión, procesos hidrológicos y grado de intensidad de estos. Son observados dichos procesos según las marcas o signos de erosión que se pueden observar en el territorio in situ como serían las grietas de desecación, cicatrices de despegue, zapamiento de la base de apoyo de la vegetación arbórea o la decapitación de la secuencia edáfica (pérdida del horizonte A).

Por último, para la obtención del mapa final que caracterice el grado de peligrosidad de los eventos de origen geomorfológico, se utilizan técnicas de evaluación cualitativa enmarcadas en el análisis multicriterio. Según esto, se ponderaría cada variable a partir del conocimiento adquirido durante el trabajo de campo anteriormente mencionado, de gabinete y la revisión bibliográfica. Los pasos a seguir en la ponderación de las variables y su normalización se obtienen a partir de los trabajos de "Evaluación de la Capacidad de Acogida" de: Ocaña Ocaña y Galacho Jiménez (2003), Galacho Jiménez y Arrebola Castaño (2008-a, 2008-b, 2013), Leyva López et al. (2009), y Galacho Jiménez et al. (2011).

Para cada puntuación se realiza una ponderación de los ítems de los mapas temáticos obtenidos, para luego ser tratados a través de una matriz por comparación (Saaty, 1980, 2008) y, ser normalizados con el cálculo de vectores y valores principales (eigenvector y eigenvalor). Como resultado final, se obtiene una cartografía de peligrosidad con criterios geomorfológicos y la descripción de sus condicionantes para la gestión del territorio relativa a un medio mediterráneo (que puede y pretende ser extrapolable a otros ámbitos semejantes). La valoración del grado de peligrosidad se define en cinco niveles distintos $(1=$ Muy poco; $5=$ Muy 
alto), zonificada por los límites de las diferentes unidades de diagnóstico y condicionada por la valoración de la ponderación de las variables en la evaluación multicriterio.

\section{RESULTADOS}

\subsection{MAPA DE UNIDADES DE DIAGNÓSTICO}

En primer lugar, se procede a la unión de los mapas temáticos de pendientes, agrupaciones litológicas, morfologías y sistemas de paisaje, cruzando dicha información con las herramientas de edición de capas vectoriales de ArcMap 10.1. De esta forma, se obtiene en una misma capa de información espacial las unidades de diagnóstico con sus respectivas características geomorfológicas (Figura 2 y Tabla 1).

Figura 2. Mapa de unidades de diagnóstico.
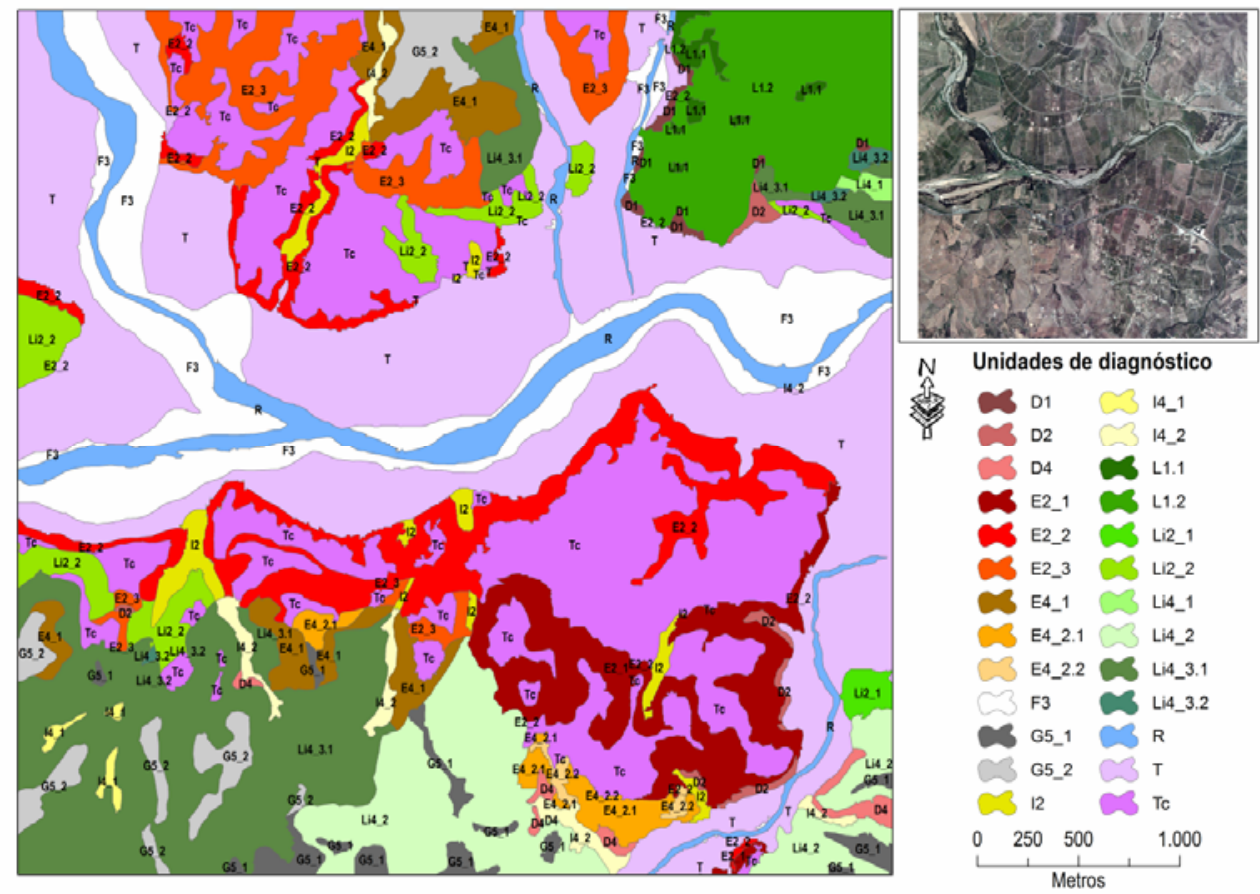

Fuente: Elaboración propia.

Así mismo, a priori, será posible apreciar diferentes condicionantes en aspectos concretos del territorio como el tipo de manto edáfico, la vegetación o los usos del suelo potenciales. 
Tabla 1. Características geomorfológicas de las unidades de diagnóstico

\begin{tabular}{|c|c|c|c|c|c|}
\hline ID & Morfología & Agrupación litológica & Pendientes (\%) & Has & $\begin{array}{c}\text { \% sobre } \\
\text { el total }\end{array}$ \\
\hline D1 & $\begin{array}{c}\text { Talud de derrubios } \\
\text { sobre materiales maláguides }\end{array}$ & $\begin{array}{c}\text { Grauwacas, calcofilitas } \\
\text { y calizas alabeadas }\end{array}$ & $21-31$ & 4,29 & 0,23 \\
\hline D2 & $\begin{array}{l}\text { Talud de derrubios sobre } \\
\text { terrazas fluviales erosionadas }\end{array}$ & $\begin{array}{c}\text { Conglomerados, gravas, arenas } \\
\text { y limos }\end{array}$ & $16-21$ & 6,69 & 0,36 \\
\hline D4 & $\begin{array}{l}\text { Talud de derrubios } \\
\text { con laderas irregulares }\end{array}$ & Gravas, arenas, limos y arcillas & $16-21$ & 7,89 & 0,42 \\
\hline E2_1 & $\begin{array}{l}\text { Escarpes y terrazas } \\
\text { fluviales erosionadas }\end{array}$ & Arenas y margas & $16-21$ & 74,4 & 3,97 \\
\hline E2_2 & $\begin{array}{c}\text { Escarpes y terrazas } \\
\text { fluviales erosionadas }\end{array}$ & $\begin{array}{c}\text { Conglomerados, gravas, arenas, } \\
\text { limos y margas }\end{array}$ & $16-21$ & 100,03 & 5,34 \\
\hline E2_3 & $\begin{array}{c}\text { Escarpes y } \\
\text { terrazas fluviales erosionadas }\end{array}$ & Arcillas, margas y areniscas & $16-21$ & 71,84 & 3,84 \\
\hline E4_1 & Escarpes con laderas irregulares & Arcillas, margas y areniscas & $16-21$ & 45,24 & 2,42 \\
\hline E4_2.1 & $\begin{array}{c}\text { Escarpes con laderas irregulares } \\
(<31 \%)\end{array}$ & $\begin{array}{c}\text { Conglomerados, gravas, arenas } \\
\text { y limos }\end{array}$ & $16-21$ & 16,37 & 0,87 \\
\hline E4_2.2 & $\begin{array}{c}\text { Escarpes con laderas irregulares } \\
(>31 \%)\end{array}$ & $\begin{array}{c}\text { Conglomerados, gravas, arenas } \\
\mathrm{y} \text { arcillas }\end{array}$ & $31-46$ & 3,04 & 0,16 \\
\hline F3 & Fondo del río y cauce arenoso & Gravas, arenas y limos & $8-16$ & 158,28 & 8,45 \\
\hline G5_1 & Glacis y superficies de erosión & Arenas y margas & $16-21$ & 22,21 & 1,19 \\
\hline G5_2 & Glacis y superficies de erosión & Arcillas, margas y areniscas & $8-16$ & 37,78 & 2,02 \\
\hline $\mathrm{I} 2$ & $\begin{array}{c}\text { Fondo de valle con terrazas } \\
\text { erosionadas }\end{array}$ & Gravas, arenas, limos y arcillas & $16-21$ & 20,7 & 1,1 \\
\hline I4_1 & $\begin{array}{c}\text { Fondo de valle con laderas } \\
\text { irregulares } \\
\end{array}$ & Arcillas, margas y areniscas & $16-21$ & 3,02 & 0,16 \\
\hline I4_2 & $\begin{array}{c}\text { Fondo de valle con laderas } \\
\text { irregulares } \\
\end{array}$ & Gravas, arenas, limos y arcillas & $16-21$ & 15,61 & 0,83 \\
\hline L1.1 & $\begin{array}{c}\text { Montaña media de } \\
\text { origen maláguide }(<31 \%)\end{array}$ & $\begin{array}{c}\text { Grauwacas, calcofilitas y calizas } \\
\text { alabeadas }\end{array}$ & $21-31$ & 6,69 & 0,36 \\
\hline $\mathrm{L} 1.2$ & $\begin{array}{c}\text { Montaña media de } \\
\text { origen maláguide }(>31 \%)\end{array}$ & $\begin{array}{c}\text { Grauwacas, calcofilitas y calizas } \\
\text { alabeadas }\end{array}$ & $46-76$ & 101,37 & 5,41 \\
\hline Li2_1 & $\begin{array}{c}\text { Terrazas fluviales erosionadas } \\
\text { sobre laderas irregulares }\end{array}$ & Arenas y margas & $21-31$ & 5,27 & 0,28 \\
\hline Li2_2 & $\begin{array}{c}\text { Terrazas fluviales erosionadas } \\
\text { sobre laderas irregulares }\end{array}$ & Arcillas, margas y areniscas & $21-31$ & 40,73 & 2,17 \\
\hline Li4_1 & $\begin{array}{c}\text { Laderas irregulares } \\
\text { sobre materiales maláguides }\end{array}$ & $\begin{array}{c}\text { Grauwacas, calcofilitas y calizas } \\
\text { alabeadas }\end{array}$ & $21-31$ & 2,57 & 0,14 \\
\hline Li4_2 & $\begin{array}{c}\text { Terrazas fluviales erosionadas } \\
\text { sobre laderas irregulares }\end{array}$ & Arenas y margas & $16-21$ & 99,48 & 5,31 \\
\hline Li4_3.1 & $\begin{array}{c}\text { Escarpes con } \\
\text { laderas irregulares }(<31 \%)\end{array}$ & Arcillas, margas y areniscas & $16-21$ & 186,66 & 9,97 \\
\hline Li4_3.2 & $\begin{array}{c}\text { Escarpes con } \\
\text { laderas irregulares }(>31 \%)\end{array}$ & Arcillas, margas y areniscas & $46-76$ & 3,02 & 0,16 \\
\hline $\mathrm{R}$ & Cauce ordinario & Gravas, arenas y limos & $3-8$ & 81,41 & 4,35 \\
\hline $\mathrm{T}$ & $\begin{array}{c}\text { Terrazas fluviales } \\
\text { no consolidadas }\end{array}$ & Gravas, arenas, limos y arcillas & $0-3$ & 469,41 & 25,06 \\
\hline $\mathrm{Tc}$ & Terrazas fluviales consolidadas & $\begin{array}{c}\text { Conglomerados, gravas, arenas } \\
\text { y limos }\end{array}$ & $8-16$ & 288,91 & 15,43 \\
\hline
\end{tabular}


Tabla 2. Sistemas morfogenéticos detectadas en las unidades de diagnóstico.

\begin{tabular}{|c|c|c|c|}
\hline ID & Marcas de erosión & Procesos & Grado \\
\hline \multirow{2}{*}{ D1 } & - horizonte A erosionado & - arroyada laminar & baja \\
\hline & - acumulación bajo las matas de vegetación & - arroyada difusa & baja \\
\hline \multirow{2}{*}{ D2 } & - horizonte A poco erosionado & - arroyada laminar & bajo \\
\hline & - acumulación bajo las matas de vegetación & - arroyada difusa & moderado \\
\hline \multirow{2}{*}{ D4 } & - horizonte A poco erosionado & - arroyada laminar & moderado \\
\hline & - acumulación en parte arriba de matas de vegetación & - agradación & moderado \\
\hline \multirow{5}{*}{ E2_1 } & - surcos y regueros de $50-500 \mathrm{~cm}$. de profundidad & - arroyada concentrada & alto \\
\hline & - túneles subsuperficiales & - humectación/desecación & alto \\
\hline & - masas desplazadas & - piping & moderado \\
\hline & - diaclasas de descompresión & - deslizamientos & moderado \\
\hline & - cicatrices de despegue & - solifluxión & alto \\
\hline \multirow{4}{*}{ E2_2 } & - surcos y regueros de $50-500 \mathrm{~cm}$. de profundidad & & \\
\hline & - diaclasas de descompresión & - arroyada concentrada & muy alto \\
\hline & - cicatrices de despegue & - desprendimientos & moderado \\
\hline & - caos de bloques al pie del escarpe & & \\
\hline \multirow{5}{*}{ E2_3 } & - surcos y regueros de $50-500 \mathrm{~cm}$. de profundidad & - arroyada concentrada & alto \\
\hline & - túneles subsuperficiales & - humectación/desecación & moderado \\
\hline & - masas desplazadas & - piping & alto \\
\hline & - diaclasas de descompresión & - deslizamientos & moderado \\
\hline & - cicatrices de despegue & - solifluxión & alto \\
\hline \multirow{4}{*}{ E4_1 } & $\begin{array}{l}\text { - surcos y regueros de } 50-500 \mathrm{~cm} . \text { de profundidad } \\
\text { - túneles subsuperficiales }\end{array}$ & $\begin{array}{c}\text { - arroyada concentrada } \\
\text { - humectación/desecación } \\
\text { - piping }\end{array}$ & $\begin{array}{l}\text { muy alto } \\
\text { alto } \\
\text { alto }\end{array}$ \\
\hline & - masas desplazadas & - deslizamientos & moderado \\
\hline & - diaclasas de descompresión & - desprendimientos & alto \\
\hline & - cicatrices de despegue & - solifluxión & alto \\
\hline \multirow{3}{*}{ E4_2.1 } & - surcos y regueros de $50-500 \mathrm{~cm}$. de profundidad & - arroyada concentrada & moderado \\
\hline & - túneles subsuperficiales & - humectación/desecación & alto \\
\hline & - masas desplazadas & $\begin{array}{c}\text { - prping } \\
\text { - deslizamientos } \\
\end{array}$ & $\begin{array}{l}\text { moderado } \\
\text { moderado }\end{array}$ \\
\hline \multirow{4}{*}{ E4_2.2 } & - surcos y regueros de $50-500 \mathrm{~cm}$. de profundidad & - arroyada concentrada & alto \\
\hline & - grietas de desecación & - humectación/desecación & alto \\
\hline & - túneles subsuperficiales & - piping & moderado \\
\hline & - masas desplazadas & - deslizamientos & muy alto \\
\hline \multirow{3}{*}{ F3 } & & - transporte de aluviones & alto \\
\hline & - barras fluviales & - erosión lateral & alto \\
\hline & & - inundación & muy alto \\
\hline \multirow{3}{*}{ G5_1 } & - horizonte A poco erosionado & - arroyada laminar & bajo \\
\hline & - insinuación de pequeños surcos & - arroyada difusa & muy bajo \\
\hline & - grietas de desecación & - humectación/desecación & bajo \\
\hline \multirow{3}{*}{ G5_2 } & - horizonte A poco erosionado & - arroyada laminar & bajo \\
\hline & - insinuación de pequeños surcos & - arroyada difusa & muy bajo \\
\hline & - grietas de desecación & - piping & moderado \\
\hline \multirow{2}{*}{ I2 } & - acumulación de derrubios finos & - agradación & bajo \\
\hline & - túneles subsuperficiales & - piping & alto \\
\hline \multirow{2}{*}{ I4_1 } & - acumulación de derrubios finos & - agradación & bajo \\
\hline & - túneles subsuperficiales & - piping & alto \\
\hline \multirow{4}{*}{ I4_2 } & - horizonte A poco erosionado & & \\
\hline & - circulación de sedimentos & - arroyada laminar & bajo \\
\hline & - acumulación de derrubios finos & - agradación & bajo \\
\hline & - túneles subsuperficiales & - piping & alto \\
\hline \multirow{3}{*}{$\mathrm{L} 1.1$} & - horizonte A erosionado & - arroyada laminar & moderada \\
\hline & - circulación de sedimentos & - arroyada difusa & alto \\
\hline & - descalzamiento de matas por debajo & - reptación & moderada \\
\hline \multirow{2}{*}{$\mathrm{L} 1.2$} & - horizonte A erosionado & - arroyada laminar & alto \\
\hline & - circulación de sedimentos & - arroyada difusa & alto \\
\hline
\end{tabular}


A partir de aquí, se reanudó el trabajo de campo y el análisis fotointerpretativo con objeto de integrar en cada unidad de diagnóstico las causas y los efectos generados por la inestabilidad de las variables anteriormente analizadas. A continuación se presenta una descripción de las marcas de erosión, los procesos geomorfológicos y el grado de intensidad (bajo, moderado, alto y muy alto) que se han visto reflejados en los suelos o la vegetación de los escarpes, las laderas, las terrazas, el lecho fluvial, los valles o los glacis, estimando también el grado de intensidad mostrado en el tiempo y en el espacio (Tabla 2).

\subsection{APLICACIÓN DE LA EVALUACIÓN MULTICRITERIO}

Definido su objeto de uso (valorar la peligrosidad con criterios geomorfológicos), la evaluación multicriterio será tomada desde la metodología descrita en Ocaña Ocaña y Galacho Jiménez (2003), Galacho y Arrebola Castaño (2008-a, 2008-b, 2013) y Galacho Jiménez et al. (2011). El resultado será una capa de información que muestre una zonificación del espacio estudiado con una puntuación asignada a cada parte del territorio según una ponderación concreta y una normalización de las variables (Galacho Jiménez et al., 2011). De esta forma, la información aparecerá ahora expresada en función del significado de la unidad de diagnóstico en su conjunto con una matriz de comparación (retocada en los trabajos mencionados anteriormente). Dicha herramienta fue denominada como matriz de puntuaciones, ya que sus valores internos son llamados puntuaciones de criterios y representan el valor o nivel de deseabilidad que se establece para cada alternativa en cada factor. Una vez que a un factor se le asignan pesos o puntuaciones, este pasa a ser un criterio (Galacho y Arrebola Castaño, 2013). Así, en la matriz, los criterios ocuparán las columnas principales y las alternativas en cada comparación las filas principales. Para la ponderación de los valores de las variables se propone el método de comparación por pares de Saaty $(1980,2008)$. El resultado es una matriz entre pares de clases, en la que se observa la importancia de cada una de ellas sobre las demás. La escala de medida establecida para la asignación de los juicios de valor es de tipo continuo (ratios o razón). Los valores se reparten desde el mínimo 1/9 hasta 9, indicando el valor 1 de igualdad en la importancia entre pares de factores (Galacho Jiménez et al. 2011). Como ejemplo, se expone cómo se ha realizado la ponderación del criterio "Agrupaciones litológicas" (Tabla 3). 
Tabla 3: Matriz de Saaty (1980) y normalización de las variables (Voogd, 1983)

\begin{tabular}{ccccccc}
\hline Ponderación de las clases & 1 & 2 & 3 & 4 & 5 & 6 \\
\hline Grauwacas, calcofilitas y calizas alabeadas (1) & 1 & 3 & 5 & 7 & 8 & 9 \\
Arcillas, margas y areniscas (2) & $1 / 3$ & 1 & 3 & 5 & 7 & 8 \\
Conglomerados, gravas, arenas y limos (3) & $1 / 5$ & $1 / 3$ & 1 & 3 & 5 & 7 \\
Arenas y margas (4) & $1 / 7$ & $1 / 5$ & $1 / 3$ & 1 & 3 & 5 \\
Gravas, arenas, limos y arcillas (5) & $1 / 8$ & $1 / 7$ & $1 / 5$ & $1 / 3$ & 1 & 3 \\
Gravas, arenas y limos (6) & $1 / 9$ & $1 / 8$ & $1 / 7$ & $1 / 5$ & $1 / 3$ & 1 \\
Totales & 1,91 & 4,80 & 9,68 & 16,53 & 24,33 & 33,00 \\
\hline
\end{tabular}

Fuente: Voogd, 1983

A continuación, "es conveniente que los valores de las alternativas en los distintos ejes estén también normalizados" (Galacho y Arrebola Castaño, 2008-a). Dicho procedimiento estadístico debe ser del tipo $0=$ mínima capacidad y $1=$ la máxima. Para este estudio, se optó por "la división de cada valor por el valor máximo" (Voogd, 1983):

$$
e_{i j}=\frac{x_{i j}-\min x_{i j}}{\max x_{i j}-\min x_{i j}}
$$

Donde: $e_{i j}$ es el valor normalizado de la alternativa $i$ en el criterio $j$. Así, $x_{i j}$ será el valor de la alternativa $i$ en el criterio $j$. Y por último, min y max comprenderán los valores mínimos y máximos de las alternativas en el criterio (Tablas 4 y 5).

Tabla 4: Normalización de las variables.

\begin{tabular}{cccccccc}
\hline \multicolumn{6}{c}{ Normalización de pesos por columnas } \\
\hline 1 & 2 & 3 & 4 & 5 & 6 & EP & EPN \\
\hline 0,52 & 0,62 & 0,52 & 0,42 & 0,33 & 0,27 & 2,69 & 0,45 \\
0,17 & 0,21 & 0,31 & 0,30 & 0,29 & 0,24 & 1,53 & 0,25 \\
0,10 & 0,07 & 0,10 & 0,18 & 0,21 & 0,21 & 0,88 & 0,15 \\
0,07 & 0,04 & 0,03 & 0,06 & 0,12 & 0,15 & 0,49 & 0,08 \\
0,07 & 0,03 & 0,02 & 0,02 & 0,04 & 0,09 & 0,27 & 0,04 \\
0,06 & 0,03 & 0,01 & 0,01 & 0,01 & 0,03 & 0,16 & 0,03 \\
1,00 & 1,00 & 1,00 & 1,00 & 1,00 & 1,00 & 6,00 & 1,00 \\
\hline
\end{tabular}

Fuente: Voogd, 1983 
Tabla 5: Normalización de las variables $\mathrm{I}^{1}$.

\begin{tabular}{cccccccc}
\hline \multicolumn{3}{l}{ Normalización de la escala de medida } & & & EP & EPN \\
\hline 1,00 & 1,00 & 1,00 & 1,00 & 1,00 & 1,00 & 5,00 & 1,00 \\
0,25 & 0,30 & 0,59 & 0,71 & 0,87 & 0,88 & 2,72 & 0,54 \\
0,10 & 0,07 & 0,18 & 0,41 & 0,61 & 0,75 & 1,37 & 0,27 \\
0,04 & 0,03 & 0,04 & 0,12 & 0,35 & 0,50 & 0,57 & 0,11 \\
0,02 & 0,01 & 0,01 & 0,02 & 0,09 & 0,25 & 0,14 & 0,03 \\
0,00 & 0,00 & 0,00 & 0,00 & 0,00 & 0,00 & 0,00 & 0,00 \\
\hline
\end{tabular}

Fuente: Voogd, 1983

Tabla 6: Ejemplo de test de consistencia: "grado de erosión".

\begin{tabular}{|c|c|c|c|c|}
\hline Grado de la erosión & Bajo & Moderado & Alto & Muy alto \\
\hline 1 & 1 & 3 & 6 & 9 \\
\hline 2 & $1 / 3$ & 1 & 3 & 6 \\
\hline 3 & $1 / 6$ & $1 / 3$ & 1 & 3 \\
\hline 4 & $1 / 9$ & $1 / 6$ & $1 / 3$ & 1 \\
\hline Sumatorio & 1,61 & 4,50 & 10,33 & 19,00 \\
\hline \multicolumn{5}{|c|}{ Porcentaje por columnas (total) } \\
\hline 1 & 0,62 & 0,67 & 0,58 & 0,47 \\
\hline 2 & 0,21 & 0,22 & 0,29 & 0,32 \\
\hline 3 & 0,10 & 0,07 & 0,10 & 0,16 \\
\hline 4 & 0,07 & 0,04 & 0,03 & 0,05 \\
\hline Vector de prioridad & 0,59 & 0,26 & 0,11 & 0,05 \\
\hline \multicolumn{5}{|c|}{ Peso del sumatorio de la matriz } \\
\hline 1 & 0,59 & 0,78 & 0,65 & 0,43 \\
\hline 2 & 0,20 & 0,26 & 0,32 & 0,29 \\
\hline 3 & 0,10 & 0,09 & 0,11 & 0,14 \\
\hline 4 & 0,07 & 0,04 & 0,04 & 0,05 \\
\hline \multicolumn{5}{|c|}{ Test de consistencia } \\
\hline 1 & 0,33 & & Lambda Max & 0,08 \\
\hline 2 & 0,00 & & $\mathrm{n}$ & 4,00 \\
\hline 3 & 0,00 & & $\mathrm{CI}$ & $-1,31$ \\
\hline \multirow[t]{2}{*}{4} & 0,00 & & RI & 1,12 \\
\hline & & & $\mathrm{CR}$ & $-1,17$ \\
\hline
\end{tabular}

Fuente: Gómez y Barredo, 2005

Estado Aceptable

${ }^{1}$ *Normalización de 1 a 0 , donde 1 = Más óptimo y $0=$ Menos óptimo 
Para controlar que la asignación de juicios de valor se ha realizado correctamente (aunque se base en criterios sólidamente establecidos, siempre se arrastra un margen importante de subjetividad), se establece el cálculo de la razón de consistencia, lo que permite reconsiderar las asignaciones realizadas en caso de inconsistencia de la matriz de comparación mediante el tratamiento estadístico de los datos con la misma hoja de cálculo (Gómez y Barredo, 2005). Esto se puede observar en un ejemplo expuesto para la ponderación asignada en el criterio de "Grado de erosión" (Tabla 6).

A continuación, se determina el eigenvector principal (EP), que representa el orden de prioridad de los factores y establecimiento de los pesos, proporcionando una medida cuantitativa de la consistencia de los juicios de valor entre pares de factores (Saaty, 1980). Para cada factor, se establece el eigenvector principal, que representará el orden de prioridad de los factores y los pesos, pasando a considerar el eigenvalor máximo (MAX 1) de la matriz como una medida de la consistencia de los juicios. Una vez realizado el proceso, conviene que sea normalizado de nuevo para obtener así el vector de prioridades y que los valores de las alternativas en los distintos ejes estén también normalizados (EPN); dicha herramienta se realiza con el criterio en el que 0 = mínima aptitud y 1 = máxima aptitud. De los múltiples sistemas de normalización de vectores se puede volver a optar por el de la división de cada valor por el valor máximo para resaltar aún más los extremos desde 0 a 1 (Voogd, 1983).

Para esta propuesta de ponderación (Tabla 7), se toma como apoyo los trabajos de van Zuidam y van Zuidam Cancelado (1979), Dent y Young (1981), CEOTMA (1984), Zinck (1988), Moreira (1991), Verstappen y van Zuidam (1991), Ferre Bueno (1997-a, 1997-b), Perles (1997). En primer lugar, para las agrupaciones litológicas y las pendientes se ha visto conveniente otorgar un peso específico (sobre un total de 1) de 0.2 y para los procesos geomorfológicos y el grado de erosión 0.25 , dándoles una mayor importancia por ser las causas más determinantes en los procesos de la peligrosidad analizada con criterios geomorfológicos (Panizza, 2005).

Con respecto a las marcas de erosión, solo se le atribuirá un 0,1 por ser el factor más subjetivo y menos definitorio de la peligrosidad, actuando solamente como un indicador pasado o potencial para el futuro (Dent y Young, 1981).

Ya cargados y normalizados en la base de datos SIG los valores EPN en la capa de unidades de diagnóstico (pendientes, agrupación litológica, marcas de erosión, procesos geomorfológicos y grados), se valora un conjunto de alternativas y un peso para cada factor. Este procedimiento es una aceptación de la compensación entre los criterios, donde el juicio personal introduce la proporción en que, según su estimación, el valor de un criterio pueda compensar el valor de otro. De esta manera, en la Tabla 8 se pueden apreciar los valores que se han obtenido como resultado de la repetición de la normalización y la ponderación final de los factores. 
Tabla 7. Ponderación de las variables ${ }^{2}$.

\begin{tabular}{|c|c|c|c|c|c|}
\hline Valores & Agrupación litológica & Pendientes & $\begin{array}{l}\text { Marcas de } \\
\text { erosión }\end{array}$ & $\begin{array}{c}\text { Procesos } \\
\text { geomorfológi- } \\
\text { cos } \\
\end{array}$ & $\begin{array}{c}\text { Grado de } \\
\text { erosión }\end{array}$ \\
\hline 1 & $\begin{array}{c}\text { Grauwacas, calcofilitas } \\
\text { y calizas alabeadas }\end{array}$ & $0-3$ y $3-8$ & $\begin{array}{l}\text { Horizonte A } \\
\text { poco erosiona- } \\
\text { do y pequeños } \\
\text { surcos, grietas y } \\
\text { acanaladuras }\end{array}$ & $\begin{array}{l}\text { Arroyada } \\
\text { laminar y difusa, } \\
\text { agradación, } \\
\text { humectación } \\
\text { desecación, } \\
\text { reptación } \\
\end{array}$ & Baja \\
\hline 2 & $\begin{array}{l}\text { Arcillas, margas y } \\
\text { areniscas }\end{array}$ & $\begin{array}{c}8-16 \\
y \\
16-21\end{array}$ & $\begin{array}{l}\text { Horizonte A } \\
\text { erosionado }\end{array}$ & $\begin{array}{l}\text { Acumulación de } \\
\text { sedimentos, } \\
\text { transporte de } \\
\text { aluviones }\end{array}$ & Moderada \\
\hline 3 & $\begin{array}{c}\text { Conglomerados, } \\
\text { gravas, arenas y limos } \\
\text { medianamente consoli- } \\
\text { dados: terrazas fluvia- } \\
\text { les consolidadas y } \\
\text { algunos taludes de } \\
\text { derrubios }\end{array}$ & $\begin{array}{c}21-31 \\
y \\
31-46\end{array}$ & $\begin{array}{l}\text { Surcos, regue- } \\
\text { ros de } 50-500 \\
\mathrm{~cm} \text {, cárcavas y } \\
\text { cicatrices de } \\
\text { despegue }\end{array}$ & $\begin{array}{c}\text { Arroyada } \\
\text { concentrada }\end{array}$ & Alta \\
\hline 4 & Arenas y margas & $\begin{array}{c}46-76 \\
y \\
>76\end{array}$ & $\begin{array}{c}\text { Acumulación } \\
\text { de sedimentos, } \\
\text { derrubios, } \\
\text { túneles subsu- } \\
\text { perficiales, } \\
\text { lecho del río y } \\
\text { barras fluviales }\end{array}$ & $\begin{array}{l}\text { Piping, desliza- } \\
\text { mientos, } \\
\text { solifluxión, } \\
\text { desprendimien- } \\
\text { tos }\end{array}$ & Muy alta \\
\hline 5 & $\begin{array}{l}\text { Gravas, arenas limos y } \\
\text { arcillas: terrazas } \\
\text { fluviales no consolida- } \\
\text { das y valles rellenados }\end{array}$ & & & $\begin{array}{l}\text { Erosión lateral e } \\
\text { inundación }\end{array}$ & \\
\hline 6 & $\begin{array}{c}\text { Gravas, arenas y limos } \\
\text { no consolidados: } \\
\text { cauces fluviales }\end{array}$ & & & & \\
\hline
\end{tabular}

Fuente: Elaboración propia.

$2 *$ Ponderación de 1 a 6 , donde $1=$ Menos peligroso y 6 = Mayor peligrosidad 
Tabla 8. Resultado final de los valores $\mathrm{EPN}^{3}$ en cada variable ponderada.

\begin{tabular}{|c|c|c|c|c|}
\hline U. de diagnóstico & $\begin{array}{c}\text { Morfometría } \\
\text { (EPN) }\end{array}$ & $\begin{array}{c}\text { Agrupaciones litológicas } \\
\text { (EPN) }\end{array}$ & $\begin{array}{c}\text { Marcas de } \\
\text { erosión (EPN) }\end{array}$ & Procesos (EPN) \\
\hline D1 & 0,13 & 1 & 0,4 & 1 \\
\hline D2 & 0,44 & 0,27 & 1 & 1 \\
\hline D4 & 0,44 & 0,03 & 1 & 1 \\
\hline E2_1 & 0,44 & 0,11 & 0,001 & 0,09 \\
\hline E2_2 & 0,44 & 0,27 & 0,001 & 0,09 \\
\hline E2_3 & 0,44 & 0,54 & 0,001 & 0,09 \\
\hline E4_1 & 0,44 & 0,54 & 0,001 & 0,09 \\
\hline E4_2.1 & 0,44 & 0,11 & 0,14 & 0,25 \\
\hline E4_2.2 & 0,13 & 0,03 & 0,14 & 0,09 \\
\hline $\mathrm{F} 3$ & 0,44 & 0,001 & 0,001 & 0,001 \\
\hline G5_1 & 0,44 & 0,11 & 1 & 1 \\
\hline G5_2 & 0,44 & 0,54 & 1 & 0,52 \\
\hline I2 & 0,44 & 0,03 & 0,4 & 0,25 \\
\hline I4_1 & 0,44 & 0,54 & 0,4 & 0,25 \\
\hline I4_2 & 0,44 & 0,03 & 1 & 0,52 \\
\hline L1.1 & 0,13 & 1 & 0,4 & 1 \\
\hline $\mathrm{L} 1.2$ & 0,001 & 1 & 0,4 & 1 \\
\hline Li2_1 & 0,13 & 0,11 & 0,001 & 0,25 \\
\hline $\mathrm{Li} 2 \_2$ & 0,13 & 0,54 & 0,001 & 0,25 \\
\hline Li4_1 & 0,13 & 1 & 0,4 & 0,52 \\
\hline Li4_2 & 0,44 & 0,54 & 0,14 & 0,09 \\
\hline Li4_3.1 & 0,44 & 0,54 & 0,14 & 0,09 \\
\hline Li4_3.2 & 0,001 & 0,54 & 0,001 & 0,09 \\
\hline $\mathrm{R}$ & 1 & 0,001 & 0,001 & 0,09 \\
\hline $\mathrm{T}$ & 1 & 0,001 & 0,14 & 0,001 \\
\hline $\mathrm{Tc}$ & 0,44 & 0,27 & 1 & 1 \\
\hline
\end{tabular}

Fuente: Elaboración propia.

${ }^{3}$ EPN (Eigen vector de prioridades normalizado) 
Tabla 9. Rangos de cada intervalo para la elaboración del mapa final.

\begin{tabular}{ccc}
\hline Rango del intervalo & Peligrosidad & Área (has) \\
\hline$<0,21$ & Muy baja & 353,18 \\
\hline $0,21-0,27$ & Baja & 23,84 \\
\hline $0,27-0,33$ & Media & 177,01 \\
\hline $0,33-0,38$ & Alta & 893,33 \\
\hline$>0,38$ & Muy alta & 425,32 \\
\hline
\end{tabular}

Fuente: Elaboración propia.

Figura 3. Mapa de peligrosidad con criterios geomorfológicos final.
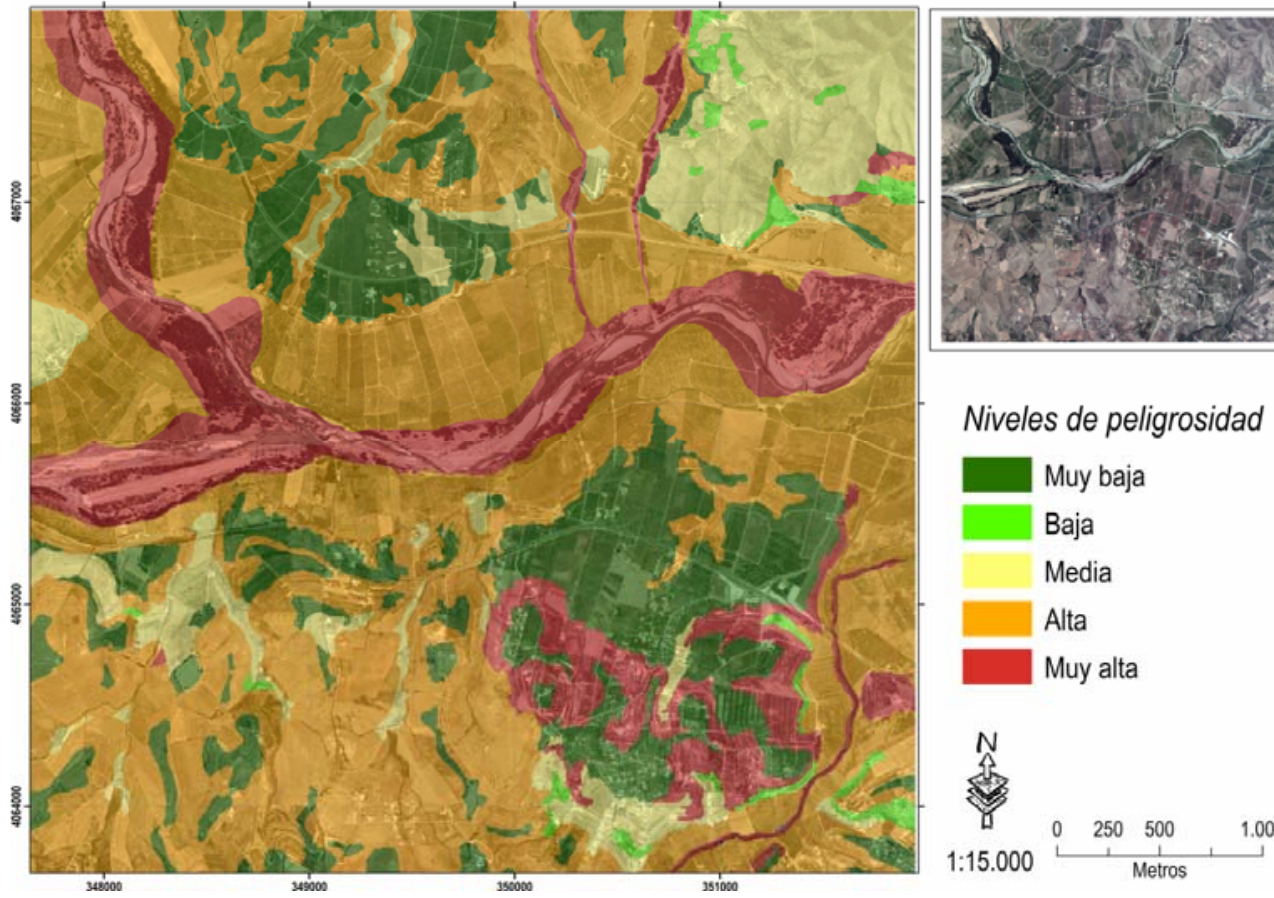

Niveles de peligrosidad
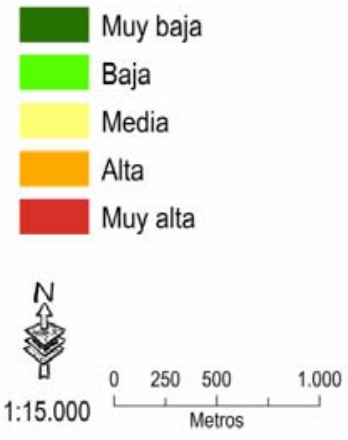

Fuente: Elaboración propia.

Para finalizar, se agrupan los datos en una serie de intervalos que sean útiles para poder diferenciar los distintos niveles de peligrosidad obtenidos (Tabla 9) y se diseña el mapa final del área de estudio (Figura 3). Para esta cartografía se utilizaron las clases de intervalos naturales (método de Jenks), basadas en las agrupaciones naturales inherentes a los datos, por ser la que mejor agrupa los intervalos sin diferenciar los valores extremos demasiado, ni centrar su importancia en los rangos 
medios de la serie. En este método, los cortes de clase se caracterizan por que agrupan mejor los valores similares y maximizan las diferencias entre clases. Las entidades se dividen en clases cuyos límites quedan establecidos donde hay diferencias considerables entre los valores de los datos. El único defecto es que no sirven para comparar varios mapas creados a partir de información subyacente distinta.

\section{CONCLUSIONES}

A través del mapa de unidades de diagnóstico se ha obtenido la zonificación de 26 espacios con características similares a una escala de trabajo de 1/25000, trasladándose también otras fuentes desde escalas más pequeñas $(1 / 50000)$, y pudiendo ser cuantificadas y cartografiadas con exactitud. Además, se ha comprobado que ha facilitado la obtención de los resultados finales en un solo mapa, la superposición de capas de información en formato vectorial apoyado en el trabajo de campo y la fotointerpretación.

A partir de aquí, es más sencillo aplicar las técnicas de análisis multicriterio para evaluar las variables cualitativas y cuantitativas. Así, se ha obtenido una zonificación final del territorio en cinco espacios con bastante exactitud, según el grado de peligrosidad estudiado con los criterios geomorfológicos asignados.

De forma general, la mayor peligrosidad del área de estudio se concentra en las áreas de influencia del cauce principal y los tributarios del río Guadalhorce. La superficie afectada por los intervalos "Alta" y "Muy alta" ocupan el 51,93\% y el $17,37 \%$ respectivamente del total analizado. Estas áreas quedan expuestas a peligros de inundación estacionales, problemas de erosión lateral (con el consiguiente peligro de generar movimientos gravitacionales de material) o depósito de sedimentos en forma de barras fluviales con un grado alto de frecuencia y potencia, conllevando potenciales obstrucciones del flujo de los cauces y aumentado los peligros por inundación.

Con una dimensión menos alarmante, aparecen las áreas catalogadas como "peligrosidad media" $(10,55 \%)$ con otra clase de problemáticas cuyo condicionante es fundamentalmente geológico. Esta problemática también afecta a las áreas más críticas anteriormente mencionadas. La razón es la inestabilidad de los materiales poco resistentes y con una elevada plasticidad por su alto contenido en arcillas expansivas. Todo esto potencia los peligros asociados al movimiento de tierras o inestabilidad de las laderas con la formación de grietas superficiales, túneles subsuperficiales y deslizamientos en masa, siendo comunes los procesos de piping.

Por otra parte, con los grados menores de peligrosidad solo se obtienen el 20,13\% del área de estudio. En estos intervalos, se hallan las áreas más alejadas del río conformadas por materiales más resistentes a la erosión de la arroyada (margas y areniscas o materiales del complejo maláguide). Pese a manifestarse sobre espacios con mayores desniveles (son comunes los intervalos entre 31-46\%), a priori, no muestran preocupantes marcas de erosión y poseen grados menores en la frecuencia de los procesos, que se podrían cuestionar a largo plazo. De esta forma, quedarían 
catalogadas como más seguras para la gestión del territorio, las terrazas fluviales consolidadas y los taludes o piedemontes compuestos por materiales del conjunto maláguide, en contraposición con los escarpes colindantes y las laderas con alto contenido de arcilla, las terrazas no consolidadas y el lecho del río ordinario. De nuevo, se observa que el condicionante geológico es definitorio en el grado de peligrosidad.

\section{DISCUSIÓN}

El motivo principal de discusión científica a raíz de este trabajo sería el planteamiento de si es posible extrapolar dicha metodología a otros espacios del área mediterránea. A la luz de los resultados obtenidos, se puede afirmar que sí. No obstante, hay que remarcar que la mayor importancia para la idoneidad del estudio, recae en la correcta realización del trabajo de campo y la fotointerpretación.

También es interesante remarcar dos puntualizaciones a discutir sobre esta metodología combinada: i) el objetivo final es la elaboración de un mapa de peligrosidad relativa a un área concreta y no de carácter absoluto para el territorio mediterráneo; ii) es un análisis de la peligrosidad de un medio "solo" desde criterios de tipo geomorfológico (Panizza et al. 1987 y Panizza, 2005).

En primer lugar, comentar que la cartografía está asociada a las condiciones de un territorio enmarcado dentro de una realidad virtualmente no reproducible en otro espacio. De ahí, que sea imprescindible, sin cambiar la secuencia metodológica, volver a escoger las variables a estudiar según el medio y la escala. Esto no debería restar importancia al trabajo como ejemplo de aplicación de una metodología extrapolable a otras áreas mediterráneas, sino, todo lo contrario, alumbrar sobre otras posibles variaciones del método.

Por otra parte, en segundo lugar, la cartografía realizada expone posibles eventos geomorfológicos extremos naturales (exclusivamente limitados a la corteza terrestre) que, asociados, pueden generar consecuencias desde un grado muy bajo a muy alto en los espacios donde se reproducen. En este trabajo no se han tratado otros posibles peligros con origen en eventos de carácter antrópico o climático, que unidos causarían efectos distintos, sin duda, sobre los elementos expuestos en el mapa final de peligrosidad de estudio (Perles Roselló et al., 2006; Cantarero Prados, 2013). De ahí que, teniendo en cuenta que las condiciones geomorfológicas constituyen uno de los principales componentes medioambientales que afectan a otros factores naturales (zonales y azonales), también se debe tener presente que representan solo una pequeña parte en la interacción de los ecosistemas (Hernández Santana et al. 2010).

Con respecto al procedimiento utilizado para el análisis (desde un SIG y la evaluación multicriterio), como afirman Ocaña Ocaña y Galacho Jiménez (2003) y Leyva López et al. (2009), "se evidencia la adecuación del área de estudio a unos usos determinados", en este caso la vocación agrícola y los espacios naturales. Sin embargo, una de las mayores ventajas es que se puede adaptar este modelo a un gran número de alternativas razonables y no quedar en un proceso cerrado: inicio en la formulación del objetivo de la valoración y fin tras la obtención de un mapa final. Su 
lógica interna radica en "una desagregación muy elevada de juicios parciales, que la convierte en una herramienta dúctil, con la que se puede interactuar, capaz de reorientar la evaluación de acuerdo a diferentes puntos de vista" (Galacho y Arrebola Castaño, 2008-a, 2013). En este sentido, tiene cabida, por tanto, experimentar sobre resultados alternativos, rectificar los juicios y considerar o no determinadas variables o criterios (Dent y Young, 1981; Triantaphyllou, 2000).

Por último, mencionar que, como se ha ido observando a lo largo de todo el trabajo, la interpretación del territorio que dio lugar al mapa de unidades de diagnóstico y los factores que conformaron los criterios de la evaluación, presentaron distintas escalas de medida, y fueron tanto atributos cualitativos, como cuantitativos. Normalmente, esto suele presentarse como un problema de base en los estudios del medio. No obstante, en esta aplicación desde las reglas de decisión, se resolvió al operar con valores numéricos a través de una misma escala y expresarlos estos en una matriz de comparación por pares.

\section{BIBLIOGRAFÍA}

AGUILAR RUIZ, J. (Coord.) et al. (1994). Mapas de suelos escala 1:50.000; hoja 1.052 (Álora); LUCDEME. Universidad de Granada.

AYALA-CARCEDO, F.J. y OLCINA CANTOS, J. (2002). Riesgos Naturales. Edit. Ariel, colección Ciencia, Barcelona.

BERTRAND, C. y BERTRAND, G. (2006). Geografía del medio ambiente. El sistema GTP: Geosistema, territorio y paisaje. Edit. Manuales de humanidades/Geografía, Universidad de Granada.

CAMACHO-OLMEDO, M.T.; PAEGELOW, M. y GARCÍA MARTÍNEZ, P. (2007). Modélisation géomatique rétrospective des paysages par évaluation multicritères et multiobjectifs. Cybergeo: European Journal of Geography, 365. URL : http://cybergeo.revues.org/4811

CANTARERO PRADOS, F. (2013). Relaciones entre el patrón territorial y la generación del peligro de inundación. Análisis comparado de casos en Málaga (España) y Motozintla (México).Tesis doctoral; Universidad de Málaga.

CASTILLO-RODRÍGUEZ, M.J.; LÓPEZ-BLANCO, E. y MUÑOZ-SALINAS (2010). A geomorphologic GIS-multivariate analysis approach to delineate environmental units, a case study of La Malinche volcano (central México). Applied Geography, 30 (4), 629-638.

CENDRERO, A. (1986). Mapa geocientífico de la provincia de Valencia. Diputación provincial de Valencia, Universidad de Valencia y Cantabria.

CEOTMA (1984). Guía para la elaboración de estudios del medio físico: contenido y metodología. Ministerio de Obras Públicas y Urbanismo, serie: Manuales.

DENT, D. y YOUNG, A. (1981). Soil Survey and Land Evaluation. Londres, George Allen y Unwin. 278 pp. 
FEIZIZADEHAB B. y BLASCHKEB, T. (2013). Land suitability analysis for Tabriz County, Iran: a multi-criteria evaluation approach using GIS. Journal of Environmental Planning and Management 56 (1), 1-23.

FERRE BUENO, E. (1997-a). Unidades de diagnóstico para la evaluación de la peligrosidad geomorfológica en el Valle del Andarax. Baetica, 19 (I), 111-134.

FERRE BUENO, E. (1997-b). Estados erosivos en la cuenca media del río Andarax. Cuadernos geográficos, 27, 153-169.

GALACHO JIMÉNEZ, F.B. y ARREBOLA CASTAÑO, J.A. (2008-a). El modelo de evaluación de la capacidad de acogida del territorio. Aspectos conceptuales y técnicas relacionadas. Baética: Estudios de arte, geografía e historia, pp. 21-40.

GALACHO JIMÉNEZ, F. B. y ARREBOLA CASTAÑO, J. A. (2008-b). Aplicación del modelo de evaluación de la capacidad de acogida para la valoración de la aptitud física del territorio respecto a sectores de planeamiento urbanístico. En: Hernández, L. y Parreño, J. M. (Eds.), Tecnologías de la Información Geográfica para el Desarrollo Territorial. Servicio de Publicaciones y Difusión Científica de la ULPGC. Las Palmas de Gran Canaria, 43-55.

GALACHO JIMÉNEZ, F. B. y ARREBOLA CASTAÑO, J. A. (2013). Modelo de evaluación de la capacidad de acogida del territorio con SIG y técnicas de decisión multicriterio respecto a la implantación de edificaciones en espacios rurales. Investigaciones Geográficas, Universidad de Alicante, 60 (2), 69-85.

GALACHO JIMÉNEZ, F. B., ARREBOLA CASTAÑO, J. A. y LUQUE GIL, A.M. (2011). Metodología de evaluación de la aptitud con relación a las infraestructuras viales ligadas a las actividades recreativas y deportivas en espacios naturales. XIII Conferencia sobre SIG (Toluca).

GÓMEZ DELGADO, M. y BARREDO CANO, J. I. (2005). Sistemas de Información Geográfica y evaluación multicriterio en la ordenación del territorio. En: Moreno, A. (2007): Sistemas y Análisis de la Información Geográfica. Manual de aprendizaje con ArcGis. Madrid, Ra Ma Editorial.

HERNÁNDEZ SANTANA, J. R.; LÓPEZ MIGUEL, C.; MÉNDEZ LINARES, A. P. y BOLLO MANNET, M. (2010). Geomorphic intensity of the northwest relief of the State of Chiapas, Mexico: an approach for territorial planning. Rev. C. y G., 24 (1-2), 79-98.

IGME (1978). Mapa geológico. E.1:50.000. Hoja 1052. Álora. Servicio de Publicaciones Ministerio de Industria, Madrid.

LEYVA LÓPEZ, J.C.; AVILÉS OCHOA, E. y ZEPEDA RODRÍGUEZ, J.J. (2009). Herramientas Operativas para el Análisis Multicriterio del Desarrollo Económico Local. Universidad de Occidente (México).

MATARREDONA, E. (1989). Aproximación a una zonación fisiográfica y su aplicación en el control de impactos ambientales, el caso de Alcoi. Investigaciones geográficas, 7, 165-190.

MOREIRA, J.M. (1991). Capacidad de uso y erosión de suelos. Una aproximación a la evaluación de tierras en Andalucía. Edit. Agencia de Medio Ambiente, Junta de Andalucía. 
OCAÑA OCAÑA, C. y GALACHO JIMÉNEZ, F.B. (2003). Aplicación de SIG y multicriterio al análisis del paisaje: cálculo y evaluación del potencial de vistas aplicado a las cuencas visuales. IX Conferencia Iberoamericana de Cáceres.

PADILLA, A.; MATARREDONA, E. y MARCO MOLINA (1998). Integración de los estudios del medio físico en la ordenación del territorio y valoración ecológicopaisajística del río Algar (Alte, Alicante). Investigaciones geográficas, 20, 119-140.

PAEGELOW, M., VILLA, N., CORNEZ, L., FERRATY, F., FERRÉ, L. y SARDA, P. (2004). Modelisations prospectives de l'occupation du sol. Le cas d'une montagne méditerranéenn. Cybergeo (Revue européenn de géographie), 295, 1-19.

PANIZZA, M. (2005). Manuale di geomorfologia applicata. Colección: Uomo, ambiente, sviluppo, Roma.

PANIZZA, M.; CASTALDINI, D.; BOLLETTINARI, G.; CARTON, A. y MANTOVANI, F. (1987). Neotectonic Research in Applied Geomorphologycal Studies. Z. Geomorph. N.F. Suppl., Bd, nº3.

PEÑA MONNÉ, J.L. (1997). Los mapas geomorfológicos. Características y tipos. En: Cartografía geomorfológica básica y aplicada. Edit. Geoforma, Universidad de Zaragoza.

PERLES, M.J. (1997). Medir la erosión: fragilidad erosiva en el valle del río Vélez. Servicio de publicaciones de la Diputación de Málaga (CEDMA), Málaga, 230.

PERLES ROSELLÓ, M.J.; CANTARERO PRADOS, F.; GALACHO, F.B.; GALLEGOS REINA, A. y VÍAS MARTÍNEZ, J.M. (2006). Propuesta metodológica para el análisis integrado de peligros asociados. Aplicación al peligro de inundación, movimientos gravitacionales y erosión hídrica. En: Actas del XII Congreso Nacional de Tecnologías de la Información Geográfica, Editorial Universidad de Granada. edic. en Cd, Granada.

PORTA J. y POCH, R.M. (2011). DPSIR analysis of land and soil degradation in response to changes in land use. Spanish Journal Soil Science $n^{\circ} 1,100-115$.

RICHTER D.D. (2007). Humanity's transformation of earth's soil: Pedology's new frontier. Soil Science. 172 (12), 957-967.

RODRIGO COMINO, J. (2012). Los suelos de la provincia de Málaga. Revisión en función de los criterios de la clasificación de FAO-WRB (2006). Memoria de Licenciatura (sin publicar), Universidad de Málaga.

SAATY, T. (1980). The Analytical Hierarchy Process. Nueva York, Editorial Mc Graw Hill.

SAATY, T. (2005). Theory and Applications of the Analytic Network Process. Pittsburgh, RWS, Publications

Saaty, T. (2008). Decision making with the analytic hierarchy process. Int. J. Services Sciences, 1 (1), 83-98.

SANZ DE GALDEANO, C. y LÓPEZ GARRIDO, A.C. (2013). Tectónica de las sierras penibéticas de Abdalajís y de Huma (provincia de Málaga, España). Su relación con el contacto con la Zona Interna. Estudios Geológicos, 69(2), 133-147.

TAPIA-VARELA, G. y LÓPEZ-BLANCO, J. (2001). Mapeo geomorfológico analítico de la porción central de la Cuenca de México: unidades morfogenéticas a escala 1:100.000. Revista de Ciencias Geológicas de México, 19 (1), 50-65. 
TRIANTAPHYLLOU, E. (2000). Multi-Criteria Decision Making Methods: A Comparative Study. Kluwer Academic Publishers. Louisiana State University. Louisiana, U. S. A.

TUGEL, A.J.; HERRICK, J.E.; BROWN, J.R.; MAUSBACH, M.J.; OUCKETT, W. y HIPPLE, K. (2005). Soil Change, Soil Survey, and Natural Resources Decision Making: A Bluepoint of Action. Soil Science Society 69, 738-747.

VAN WESTEN, C.J.; RENGERS, N. y SOETERSB, R.(2003). Use of Geomorphological Information in Indirect Landslide Susceptibility Assessment. Natural Hazards, 30 (3), 399-419.

VAN ZUIDAM, R.A. y VAN ZUIDAM CANCELADO, F.I. (1979). Terrain analysis and classification using aerial photographs. A geomorphologycal approach. ITC, Textbook of Photo-Interpretation VII-6, Enschede (Países Bajos).

VERSTAPPEN, H. y VAN ZUIDAM, R.A. (1991). The ITC system of geomorphologic survey: a basis for the evaluation of natural resources and hazards. ITC Publication, 10.

VOOGD, H. (1983). Multicriteria Evaluation for Urban and Regional Planning. Londres, Pion.

ZINCK, J.A. (1988). Soil Survey Courses. ITC, Enschede.

ZINCK, J.A. (2012). Geopedología: Elementos de geomorfología para estudios de suelos y de riesgos naturales. ITC, Enschede. 